American Journal of Animal and Veterinary Sciences 5 (3): 192-195, 2010

ISSN 1557-4555

(C) 2010 Science Publications

\title{
Beneficial Effect of a Cellulose-Containing Chew Treat on Canine Periodontal Disease in a Double-Blind, Placebo-Controlled Trial
}

\author{
${ }^{1}$ A.C. Beynen, ${ }^{2}$ F. Van Altena and ${ }^{2}$ E.A. Visser \\ ${ }^{1}$ Vobra Special Petfoods BV, Veghel, The Netherlands \\ ${ }^{2}$ University of Applied Sciences Van Hall Larenstein, Leeuwarden, The Netherlands
}

\begin{abstract}
Problem statement: There are indications that appropriate chew treats can contribute to the control of canine periodontal disease. It was reasoned that the incorporation of a cellulose fiber network into the treat may improve the efficacy, but for proof experimental data were required. Approach: A double-blind, placebo-controlled trial with privately owned dogs was carried out to assess the efficacy of a cellulose preparation (Arbocel BWW40®) in the treatment of periodontal disease. With the use of a questionnaire, the clinical signs were evaluated by the owners. There were 10 clinical signs: extent and severity of dental plaque and calculus, extent of gingivitis, redness, swelling, bleeding and firmness of gingivae and halitosis. For a period of 8 weeks, the test dogs daily received a chew treat to which $4 \%$ of the cellulose preparation was added. The control dogs were given a chew treat with identical formula, but without added cellulose. During the trial, all dogs were fed the same, complete dry food. There were 16 test dogs and 15 control dogs. Results: When compared with the baseline values, the administration of the test chew significantly improved 8 out of the 10 clinical signs. In the placebo group there was a significant improvement for 6 clinical signs. When the improvements over time for the two groups were compared, there were no statistically significant differences. When the score changes for all 10 clinical signs were added up as an overall index of improvement of periodontal disease, the test group showed a $17 \%$ greater amelioration than did the control group. Conclusion: The addition of the cellulose preparation had further enhanced the efficacy of the treat, possibly through an increase in mechanical cleansing and chewing time. This study indicates that a cellulose-containing treat is beneficial for dogs with periodontal disease and it is suggested that it may also impair its development.
\end{abstract}

Key words: Placebo group, periodontal disease, cellulose preparation, irritated or inflamed, plaque, molars, gingivae, chew treat

\section{INTRODUCTION}

Periodontal disease is a common disease in adult dogs and thus represents a frequent diagnosis in veterinary practice. The disease is initiated by dental plaque formation consisting of aggregates of bacteria, salivary glycoproteins and oral debris of different origin. The dental plaque then mineralizes into calculus. The next stage is inflammation of the gingivea which can ultimately lead to destruction of the periodontium and tooth mobility.

The consistency and composition of diet kibbles play an important role in the development and treatment of canine periodontal disease (Beynen, 2008). When a dog chews the kibble, it should not crumble, but stay together so as to maintain contact with the tooth surface and provide mechanical cleansing (Logan et al., 2000). In addition, the outer layer of the kibble may contain nutraceuticals that have anti-bacterial activity (Isogai et al., 1995) or contain an inorganic polyphosphate to bind calcium and thereby reduce calculus accumulation (Stookey et al., 1995). Dog owners generally favor the administration of a treat with the claim of dental benefits rather than the feeding of a complete dental food. There is evidence that rawhide strips and boneshaped treats based on rice and whey contribute to the control of calculus formation (Lage et al., 1990; Gorrel and Rawlings, 1996).

The Arbocel BWW40® preparation of cellulose (Rettenmaier and Söhne GmbH + Co KG, Rosenberg, Germany) is a natural, highly purified product derived from different wood sources. The cellulose preparation forms a completely insoluble fiber network. Given the physical properties of the cellulose preparation it would be anticipated that its addition to a chew treat may promote a smooth, mechanical cleansing of teeth. This

Corresponding Author: A.C. Beynen, Vobra Special Petfoods BV, Veghel, The Netherlands 
study addresses the efficacy of the cellulose preparation in the treatment of canine periodontal disease. As in previous intervention studies (Beynen and Legerstee, 2010; Beynen et al., 2010), privately owned dogs were used in a double-blind, placebo-controlled trial and the clinical signs were evaluated by the owners. For a period of 8 weeks, the dogs consumed the same, complete dry food and also daily received a chew treat without or with $4 \%$ of the cellulose preparation.

\section{MATERIALS AND METHODS}

Animals: Dogs with signs of periodontal disease were recruited through the websites of breed associations and dog fancier clubs and newsletters of veterinarians. The (potential) participants were informed about the purpose and design of the trial and had to sign a statement on informed consent. In total, 42 dogs were subjected to either the placebo or test group. Eleven dogs did not finish the trial for various reasons so that the data for 31 dogs ( 15 control and 16 test dogs) were available for analysis. Table 1 shows the characteristics of the dogs as based on the intake questionnaire that was completed by their owners.

Experimental design: Recruitment of the dogs, maintaining contact with the dog owners, supplying of food and treats, data collection and general coordination of the trial was done by FvA and EAV who were blinded to treatment modality. The eligible dogs were allocated to either the placebo or treatment group by $\mathrm{ACB}$, who kept the treatment code closed until statistical analysis of the data. Allocation was done so that the distribution of body weights and the extent and severity of dental plaque, as based on the intake questionnaire, would be similar among the two groups. All dogs were fed on the same, complete dry food (Carocroc Chicken and Rice 23/12, Vobra Special Petfoods BV, Veghel, The Netherlands), which was supplied in $20-\mathrm{kg}$, blank packaging. The extruded control and test treats were produced by Rondo Food GmbH \& Co. KG, Krefeld, Germany. The control treat was grain based, contained no added cellulose and had the following composition according to the manufacturer: crude protein, $16 \%$; crude fat, $3 \%$; crude fiber, $2 \%$; crude ash, $9 \%$, moisture, $17 \%$. The test treat was made by adding $4 \%$ of Arbocel BWW40® to the control formula. The transversal cut of the treats had a star form. The weight of a single treat was about $26 \mathrm{~g}$ and the length was $15 \mathrm{~cm}$. The treats were packed in closed, blank plastic bags each containing 7 pieces. The foods and treats were sent by courier to the dog owners. The trial lasted 10 weeks. The first two weeks served as a baseline. During the second week the dogs were gradually transferred from their habitual diet to the food supplied. As from the third week, one treat daily was administered for another 8 weeks.

Trial questionnaire: The trial questionnaire was in the form of a booklet, which also provided instructions, including a completed example of a question in the format used. The booklet was sent to the dog owners together with the food and treats. The degree of the signs of dental disease was scored by the owners by signing a cross on a horizontal line. The line was without any unit, but functioned as a scale in combination with the description. The line had a length of $100 \mathrm{~mm}$. The owners scored the extent and/or severity of the following signs: plaque, calculus, gingivitis, redness, swelling, bleeding and firmness of gingivae and halitosis. The signs were scored on day 0 (start) and weekly afterwards.

To aid in scoring the signs, the following descriptions were given.

Extent of dental plaque: "Dental plaque is a lightcolored, soft-like, 1-2 mm thick, sometimes flaky layer, that can be easily removed". On how many teeth and molars is plaque present? The scale ran, from "Almost all teeth/molars" (extreme left) to "Very few teeth/molars" (extreme right).

Severity of dental plaque: "Are the teeth/molars only covered with plaque on the edges or is almost the whole surface of the teeth/molars covered?" The scale ran, from "Almost completely covered" (extreme left) to "Almost not covered at all" (extreme right).

Extent of dental calculus: "Dental calculus is hardened plaque and can be present above or beneath the gums. On how many teeth/molars does your dog have calculus?" The scale ran from "Almost all teeth/molars to "Very few teeth/molars" (extreme right).

Table 1: General characteristics of the dogs

\begin{tabular}{lll}
\hline Characteristic & Placebo treat $(\mathrm{n}=15)$ & Cellulose treat $(\mathrm{n}=16)$ \\
\hline Mean age, years (range) & $8.3(2-14)$ & $7.3(3-11)$ \\
Mean body weight, kg (range) & $18.25(4.3-34.1)$ & $14.84(4.2-38.0)$ \\
Gender, female/male & $8 / 7$ & $7 / 9$ \\
Previous professional dental cleaning, yes/no & $6 / 9$ & $4 / 12$ \\
\hline
\end{tabular}


American J. Animal \& Vet. Sci., 5 (3): 192-195, 2010

Table 2: Group mean baseline values and changes over time in the clinical signs of periodontal disease (improvement is indicated by a + sign)

\begin{tabular}{|c|c|c|c|c|c|c|c|}
\hline \multirow[b]{2}{*}{ Variable } & \multicolumn{3}{|c|}{ Placebo treat $(\mathrm{n}=15)$} & \multicolumn{3}{|c|}{ Cellulose treat $(\mathrm{n}=16)$} & \multirow{2}{*}{$\begin{array}{l}\text { p-value for } \\
\text { between-group } \\
\text { difference in change } \\
\text { (2-tailed) }\end{array}$} \\
\hline & Baseline & $\begin{array}{l}\text { Change versus } \\
\text { baseline (range) }\end{array}$ & $\begin{array}{l}\text { p-value for } \\
\text { change (2-tailed) }\end{array}$ & Baseline & $\begin{array}{l}\text { Change versus } \\
\text { baseline (range) }\end{array}$ & $\begin{array}{l}\text { p-value for } \\
\text { change (2-tailed) }\end{array}$ & \\
\hline Extent of plaque & 49.5 & $+13(-3-+37)$ & 0.001 & 50.8 & $+11(-23-+59)$ & 0.066 & 0.855 \\
\hline Severity of plaque & 58.1 & $+13(-7-+33)$ & 0.001 & 60.1 & $+9(-10-+55)$ & 0.037 & 0.519 \\
\hline Extent of calculus & 58.3 & $+7(-13-+25)$ & 0.087 & 49.6 & $+13(-8-+59)$ & 0.008 & 0.322 \\
\hline Severity of calculus & 61.1 & $+4(-18-+25)$ & 0.209 & 56.8 & $+9(-32-+62)$ & 0.090 & 0.485 \\
\hline Extent of gingivitis & 69.1 & $+13(0-+51)$ & 0.008 & 65.5 & $+10(-5-+42)$ & 0.005 & 0.637 \\
\hline Redness of gingivae & 65.2 & $+10(0-+28)$ & 0.005 & 59.9 & $+9(-14-+44)$ & 0.038 & 0.786 \\
\hline Swelling of gingivae & 76.4 & $+6(-10-+27)$ & 0.023 & 67.7 & $+11(-12-+46)$ & 0.026 & 0.798 \\
\hline Bleeding of gingivae & 85.9 & $+7(-9-+53)$ & 0.136 & 68.7 & $+10(-4-+52)$ & 0.044 & 0.781 \\
\hline Firmness of gingivae & 76.1 & $+2(-18-+22)$ & 0.542 & 68.8 & $+8(-10-+44)$ & 0.034 & 0.190 \\
\hline Halitosis & 52.0 & $+14(-2-+66)$ & 0.007 & 55.5 & $+14(-3-+54)$ & 0.005 & 0.826 \\
\hline
\end{tabular}

Severity of dental calculus: "Are the teeth/molars only covered with calculus on the edges or is almost the whole surface of the teeth/molars covered?" The scale ran from "Almost completely covered" (extreme left) to "Almost not covered at all" (extreme right).

Gums inflammation: "Gums inflammation is typified by redness, easy bleeding, swelling and lack of firmness around the teeth". Around how many teeth/molars does your dog show signs of gums inflammation?" The scale ran from "Almost all teeth/molars" (extreme left) to "Very few teeth/molars" (extreme right).

Redness of gums: "How red are the gums of your dog at places where they are irritated or inflamed?" The scale ran from "Very red" (extreme left) to "Not red" (extreme right).

Swelling of gums: "What is the degree of swelling of the gums of your dog at places where they are irritated or inflamed? The scale ran from "Very swollen" (extreme left) to "Not swollen" (extreme right).

Bleeding of gums: "Do the gums, at places where they are irritated or inflamed, bleed upon touching?" The scale ran from "Rapid bleeding" (extreme left) to "No bleeding" (extreme right).

Firmness of gums: "How firm are the gums around the teeth at places where they are irritated or inflamed?" The scale ran from "Not firm" (extreme left) to "Very firm" (extreme right).

Bad breath: "To what extent do you experience malodor from the mouth of your dog?" The scale ran from "Unbearable malodor" (extreme left) to "No malodor" (extreme right).

Data analysis: After scoring by the owner of the clinical signs in the booklet, the crosses on the lines were expressed as $\mathrm{mm}$ distance from the extreme left. Thus, the extreme left was identical to $0 \mathrm{~mm}$ and the extreme right to $100 \mathrm{~mm}$. The values are reported here without unit. To calculate the baselines, the values for day 0 and weeks 1 and 2 were averaged per variable per dog. To calculate the final values, those for weeks 9,10 and 11 were averaged. For each dog and each variable, the change over time was calculated. To identify significant changes over time for the placebo and test group, the values were subjected to the Student's t test for normally distributed data and to the Wilcoxon rank sum test for non-normally distributed values. To compare the changes between the two groups, the Student's $t$ test and Mann-Whitney U test were used for normally and non-normally distributed values, respectively. Throughout, $\mathrm{p}<0.05$ was preset as criterion of statistical significance.

\section{RESULTS}

Table 1 show that the general characteristics of the placebo and test group were similar. The baseline values for the clinical signs of periodontal disease were comparable for the test and placebo group, except for the extent of dental calculus, swelling of gingivae and bleeding of gingivae (Table 2). In the control dogs, the three signs were less severe than in the test group.

When compared to the baseline values, the administration of the control treat was associated with a significant improvement for 6 out of the 10 clinical signs (Table 2). In the dogs given the treat containing cellulose, there was a significant improvement for 8 out of the 10 clinical signs of periodontal disease. The changes over time were added up for each group to arrive at an overall index of improvement of periodontal disease. The index was 89 for the placebo group and 104 for the test group. The extra improvement caused by the treat containing cellulose was $17 \%$. When the changes over time of the two groups were compared, there were no statistically significant differences.

\section{DISCUSSION}

To test whether the incorporation of a specified cellulose preparation into a chew treat will improve canine periodontal disease, the double-blind, clinical trial was appropriate. The double-blind nature of the trial did exclude any observer bias. The general 
characteristics of the two treatment groups were similar which would nullify any group bias, but three clinical signs at baseline were more severe in the test group. Possibly, the group difference at baseline had partly caused the more positive response of the extent of dental calculus, swelling of gingivae and bleeding of gingivae in the test group. As from the start of the trial, the control and test dogs were fed on the same diet which excluded any diet difference between the two groups. It could be argued that the food rather than the treats had improved the clinical signs of periodontal disease over time. However, this is unlikely because the diet used did not have the typical properties of a dental diet as described below. In any event, when comparing the two treatments any effects of diet, season and observer attitude will be taken into account.

In order to claim that the incorporation of the cellulose preparation into the chew treat will improve canine periodontal disease, the chew treat with the cellulose should have provoked a significantly better effect than the placebo in the form of the same treat, but without the cellulose. This prerequisite is not unequivocally met by the present trial. In the course of the trial, the test versus placebo group showed a 17\% greater overall index of the improvement of periodontal disease. Furthermore, the test treat significantly improved over time 8 out of the 10 clinical signs, whereas the control treat significantly improved 6 signs. In the light of the systematic, positive, numerical effects of the cellulose-containing treat, it could be suggested that the lack of statistical significance between the group differences in the changes over time is caused by insufficient statistical power rather than by an inefficacy of the test treat.

The positive effect of the cellulose preparation on the clinical signs of periodontal disease may relate to an increase in mechanical cleansing of teeth and in chewing time. The insoluble fiber network created by the cellulose may diminish shattering and crumbling of the treat while being penetrated by the teeth. It may thus promote chewing and prolong contact with the tooth surface. Unfortunately, chewing time was not measured in the present study. The effects of foods with low or high elasticity and resistance against crumbling have been compared as to efficacy against periodontal disease (Logan et al., 2000). In dogs with plaque index of zero and clinically healthy gingivae, the increase with time in plaque formation and gingivitis development were depressed by the dental food with high elasticity and resistance against crumbling. In dogs with existing plaque, calculus and gingivitis, the dental food reduced the severity of periodontal disease, whereas it generally progressed when the control diet was fed. These data point at the importance of mechanical tooth cleansing in the control of canine periodontal disease.

\section{CONCLUSION}

This study indicates that a cellulose-containing treat versus a treat without cellulose further diminished the clinical signs in dogs with periodontal disease. The beneficial effect of the treat with cellulose did not reach statistical significance, but it appears clinically relevant. The positive effect of the cellulose preparation under study may relate to an increase in mechanical cleansing of teeth and chewing time. Based on the outcome of studies with a dental food (Logan et al., 2000), it can be suggested that the cellulose-containing treat not only diminishes the severity of existing periodontal disease, but also reduces the development.

\section{REFERENCES}

Beynen, A.C., 2008. Optimalisering van een dieetvoeder voor honden met parodontale aandoeningen. Dier-en-Arts, 23: 232-237.

Beynen, A.C. and E. Legerstee, 2010. Influence of dietary beta-1,3/1,6-glucans on clinical signs of canine osteoarthritis in a double-blind, placebocontrolled trial. Am. J. Anim. Vet. Sci., 5: 97-101. DOI: $10.3844 / .2010 .97 .101$

Beynen, A.C., H.W. Van Geene, H.V. Grim, P. Jacobs and T. Van der Vlerk, 2010. Oral administration of gelatin hydrolysate reduces clinical signs of canine osteoarthritis in a double-blind, placebo-controlled trial. Am. J. Anim. Vet. Sci., 5: 102-106. DOI: $10.3844 / .2010 .102 .106$

Gorrel, C. and J.M. Rawlings, 1996. The role of a tooth-brushing and diet in the maintenance of periodontal health in dogs. J. Vet. Dent., 13: 139-143. PMID: 9520789

Isogai, E., H. Isogai, K. Kimura, T. Nishikawa and N. Fujii et al., 1995. Effect of Japanese green tea extract on canine periodontal diseases. Microbiol. Ecol. Health Dis., 8: 57-61. DOI: 10.3109/08910609509141383

Lage, A., N. Lausen, R. Tracy and E. Allred, 1990. Effect of chewing rawhide and cereal biscuit on removal of dental calculus in dogs. J. Am. Vet. Med. Assoc., 197: 213-219. PMID: 2384322

Logan, E.I., R.B. Wiggs, K. Zetner, J.J. Hefferren, 2000. Dental disease. In: Small Animal Clinical Nutrition, Hand, M.S., C.D. Thatcher, R.L. Remillard, P. Roudebush and L.D. Lewis et al. (Eds.). Mark Morris Institute, Marceline, MI., ISBN: 10: 0945837054, pp: 475-504.

Stookey, G.K., J.M. Warrick and L.L. Miller, 1995. Effect of hexametaphosphate on dental calculus formation in dogs. Am. J. Vet. Res., 56: 913-918. PMID: 7574160 